\title{
PERSISTÊNCIA INFLACIONÁRIA REGIONAL BRASILEIRA: UMA APLICAÇÃO DOS MODELOS ARFIMA
}

\author{
Cleomar Gomes da Silva * \\ Flávio Vilela Vieira ${ }^{\dagger}$
}

\begin{abstract}
Resumo
Este artigo analisa o fenômeno da persistência das taxas de inflação (IPCA) das regiões metropolitanas de Belém, Fortaleza, Recife, Salvador, Belo Horizonte, Rio de Janeiro, São Paulo, Curitiba e Porto Alegre, além de Brasília e Goiânia. São utilizados Modelos Autorregressivos de Integração Fracionária (ARFIMA) e testes com quebras estruturais para o período de agosto de 1999 a dezembro de 2011. Os resultados mostram certa persistência inflacionária nas regiões brasileiras, mas com características de estacionariedade e reversão à média de longo prazo. Entretanto, tal resultado somente é alcançado após o tratamento das quebras estruturais, que influenciaram principalmente os resultados do Rio de Janeiro e do Recife.
\end{abstract}

Palavras-chave: Persistência Inflacionária; Política Monetária; Modelos ARFIMA.

\begin{abstract}
This paper analyzes the degree of persistence of the Broad Consumer Price Index (IPCA) of the following Brazilian regions: Belém, Fortaleza, Recife, Salvador, Belo Horizonte, Rio de Janeiro, São Paulo, Curitiba, Porto Alegre, Brasília and Goiânia. Comparisons among the regional indices and the Aggregate IPCA were made. Auto-Regressive Fractionally Integrated (ARFIMA) models and tests with structural breaks were used for the period ranging from August 1999 to December 2011. We found that inflation rates in the Brazilian regions have some degree of persistence, but they are stationary and mean-reverting. This result was only achieved when structural breaks were accounted for, once they influenced particularly the results of Rio and Recife.
\end{abstract}

Keywords: Inflation Persistence; Monetary Policy; ARFIMA Models.

JEL classification: C22, E31, E52

\footnotetext{
* Instituto de Economia - Universidade Federal de Uberlândia e CNPQ.

E-mail: cleomargomes@ie.ufu.br

† Instituto de Economia - Universidade Federal de Uberlândia, CNPQ e FAPEMIG.

E-mail: flaviovieira@ufu.br
} 


\section{Introdução}

Uma das questões fundamentais acerca da dinâmica dos preços da economia está relacionada ao comportamento inercial da inflação ou, em outras palavras, ao fenômeno da persistência inflacionária. Em linhas gerais, tal fenômeno pode ser definido como a propensão de a inflação convergir lentamente à meta, por conta da influência dos preços defasados. Ele também pode ser visto como uma correlação serial positiva na inflação ${ }^{1}$ e manifesta simultaneamente o processo de precificação dos produtos, a condução de política monetária e a formação de expectativas dos agentes econômicos (Batini 2006).

Ainda que o comportamento inercial da inflação tenha decrescido em vários países na última década, principalmente em função da condução de política monetária, ele ainda continua sendo detectado. O que se busca, entretanto, é que tal comportamento seja minimizado ao máximo no intuito de se ter uma condução de política monetária que, ao final, imponha uma taxa de sacrifício cada vez menor para a população.

A análise da persistência inflacionária não é menos importante no Brasil, pois tendo passado por um amplo processo de desinflação a partir da instituição do Plano Real, o país ainda guarda consigo resquícios de inércia nos preços que podem ser detectados nos seguintes processos indexadores, por exemplo: 1) índices de preços de contratos de concessões de energia elétrica; 2) contratos de aluguel; 3) regra do salário mínimo, dentre outros.

Mas esse comportamento não é algo restrito ao índice de preços nacional, ainda que o IPCA seja o balizador das decisões de política monetária. A dinâmica de preços também adquire características específicas em cada região do Brasil. Assim, o objetivo deste artigo é analisar o fenômeno da persistência inflacionária referente ao Índice de Preços ao Consumidor (IPCA) das regiões metropolitanas de Belém, Fortaleza, Recife, Salvador, Belo Horizonte, Rio de Janeiro, São Paulo, Curitiba e Porto Alegre, além de Brasília e do município de Goiânia. Comparações são feitas entre os índices regionais e o IPCA nacional. Para uma análise que compreende o período de agosto de 1999 a dezembro de 2011 são utilizados Modelos Autorregressivos de Integração Fracionária (ARFIMA), assim como testes de raiz unitária com quebras estruturais. Os resultados mostram que ainda existe certo grau de persistência inflacionária nas regiões brasileiras, mas com características de estacionariedade e com rápida reversão à média de longo prazo. Entretanto, tal resultado somente é conseguido para todas as localidades após o tratamento das quebras estruturais, que influenciaram principalmente as taxas de inflação referentes às regiões metropolitanas do Rio de Janeiro e do Recife.

Além desta introdução, este artigo apresenta, na segunda seção, a literatura pertinente ao tópico. A terceira seção traz o arcabouço econométrico dos modelos ARFIMA e das quebras estruturais. A quarta seção detalha os dados e a quinta reporta os resultados. A última seção faz a conclusão do trabalho.

\section{A Literatura}

Artigos importantes sobre persistência da inflação foram publicados ao longo dos últimos anos, com o uso de diversas técnicas econométricas. Dentre eles podem ser citados os trabalhos de Fuhrer \& Moore (1995), para os EUA; de

\footnotetext{
${ }^{1}$ Batini (2006) caracteriza mais outros dois tipos de persistência.
} 
Batini \& Nelson (2001), para o Reino Unido; de Gadzinski \& Orlandi (2004), para a União Europeia e EUA; de Batini (2006), também para a União Europeia. Todos eles encontram casos moderados de persistência inflacionária.

Com relação aos Modelos ARFIMA, especificamente, Doornick \& Ooms (2004) analisam os casos dos EUA e do Reino Unido. Para o primeiro, a amostra tem periodicidade trimestral (1957:01 a 2003:04) e os parâmetros fracionados ficam em torno de 0,32 , mostrando que a série pode ser descrita como estacionária. Para o caso britânico, a amostra tem periodicidade trimestral (1959:1 a 2002:2) e as estimações dos parâmetros fracionados variam entre 0,47 e 0,59, sinalizando uma provável não estacionariedade da série, mas convergência a uma média de longo prazo.

Gil-Alana (2005) aplica a metodologia ARFIMA para a taxa de inflação dos EUA e conclui que os resultados variam consideravelmente, dependendo da forma como as perturbações $\mathrm{I}(0)$ são especificadas no modelo. Por exemplo, para a especificação com ruído branco, as regressões mostram uma inflação americana estacionária com o parâmetro fracionado igual a 0,25.

Em relação ao estudo do processo inflacionário brasileiro, Cati et al. (1999) analisam o período de janeiro de 1974 a junho de 1993, caracterizado por uma grande influência do efeito da implementação de vários planos de estabilização. Essas intervenções governamentais abruptas agiram como inliers, pois a inflação era reduzida apenas temporariamente e retornava à sua trajetória inicial algum tempo depois. Os autores utilizam testes padrões de raiz unitária e reportam, como resultado, que as séries são estacionárias e que as perturbações observadas possuem somente efeitos temporários. Contudo, quando estatísticas mais apropriadas são usadas, para que sejam levados em conta os planos de estabilização, os resultados mostram que o comportamento estocástico da taxa de inflação brasileira no período analisado é, de fato, muito instável. Dessa maneira, a interpretação macroeconômica para os resultados encontrados para o período analisado está em linha com a hipótese da inércia inflacionária (Arida \& Lara-Rezende 1985, Bresser-Pereira \& Nakano 1986, dentre outros), que afirmaram que as perturbações inflacionárias da época eram extremamente persistentes.

Tejada \& Portugal (2001) investigam os efeitos da credibilidade dos Planos Cruzado, Collor e Real. O resultado é o esperado: a credibilidade afeta o comportamento da taxa de inflação e, consequentemente, sua inércia. $\mathrm{Na}$ análise dos três planos, somente o Plano Real foi capaz de reduzir a taxa de inflação a níveis baixos por um longo período de tempo, diminuindo o grau de inércia inflacionária da economia de forma consistente.

Campêlo \& Cribari-Neto (2003) mostram que testes de raiz unitária robustos podem gerar a mesma inferência sobre a ordem de integração das séries, sem a necessidade do emprego de variáveis dummy que levem em conta a presença de inliers. Os autores usam duas séries mensais distintas relativas à taxa de inflação brasileira. A primeira delas é similar àquela utilizada por Cati et al. (1999) e a segunda compreende os períodos entre fevereiro de $1944 \mathrm{e}$ fevereiro de 2000. O resultado principal aponta para a presença de inércia inflacionária que, ao contrário de trabalhos anteriores, se mostra pequena.

Yoon (2003) também utiliza os dados de Cati et al. (1999) para estimar um teste de raiz unitária proposto por Ng \& Perron (2001), que constroem quatro estatísticas de teste baseadas no procedimento detrending GLS. Com essa técnica, é possível chegar às mesmas conclusões de Cati et al. (1999), sem a ne- 
cessidade do uso de variáveis dummy. O autor conclui que a taxa de inflação não é estacionária para o período analisado.

Cribari-Neto \& Cassiano (2005) propõem o uso de uma medida robusta do impacto de choques econômicos no longo prazo para a análise do IGP-DI brasileiro, abrangendo o período de fevereiro de 1944 a junho de 2003. Os resultados empíricos sugerem que a componente inercial na dinâmica da inflação no Brasil é maior do que aquela encontrada por Campêlo \& Cribari-Neto (2003), estando em concordância com os resultados de Cati et al. (1999). Além disso, os resultados mostram que após o Plano Real a dinâmica da inflação adquiriu um caráter quase estacionário.

Especificamente em relação à aplicação dos Modelos ARFIMA para o caso brasileiro, um dos estudos pioneiros foi desenvolvido por Baillie et al. (1996), para o índice de preços mensal no período de 1957 a 1991. Levando em conta questões de volatilidade, via ARFIMA-GARCH, o resultado final indica a não estacionariedade da taxa de inflação brasileira $(d=0,598)$.

Reisen et al. (2003) investigam a existência de memória longa na dinâmica da inflação brasileira a partir de dados do IGP-DI, de fevereiro de 1944 a fevereiro de 2000. As estimações dos modelos ARFIMA indicam que a ordem de integração encontrada é menor que 1 , ou seja, há inexistência de inércia inflacionária no país, mas sim uma dinâmica inflacionária mais bem modelada por um processo de memória longa. Os choques inflacionários demoram a se dissipar, mas isso acaba ocorrendo, indicando a não persistência de tais perturbações.

Figueiredo \& Marques (2009) estudam o fenômeno da persistência na inflação brasileira para dados mensais do IGP-DI de janeiro de 1980 a janeiro de 2008. A estimação dos modelos ARFIMA foi restrita às subamostras 1980:011991:06 e 1994:08-2008:01 e indica que as séries são estacionárias, dado que os valores dos parâmetros ' $d$ ' são inferiores a 0,5 . A estimativa do modelo ARFIMA-FIGARCH, capaz de detectar a memória de curto e longo prazos e o comportamento não linear da série de inflação, foi restrita à subamostra 1994:08-2008:01 em função dos problemas relacionados à presença de outliers e inliers. Os resultados indicam que a taxa de inflação exibe comportamento estacionário e pode ser descrita como um processo fracionário de memória longa.

Figueiredo \& Marques (2011) analisam a dinâmica da inflação brasileira com dados mensais do IGP-DI de fevereiro de 1944 a agosto de 2009, utilizando modelos MS-ARFIMA, capazes de captar a mudança de regime e a presença de memória longa em altas defasagens. A estimação inicial dos parâmetros fracionários varia entre 0,72 e 0,93 , apontando para um comportamento não estacionário com reversão à média. Ao adotarem um modelo MS-ARFIMA $(1, d, 1)$, os autores encontram dois regimes distintos para a economia brasileira. O primeiro regime (anos 1960 e 1980) revela a existência de indexação plena com uma trajetória explosiva para a taxa de inflação e com altíssima volatilidade. O segundo regime (início da industrialização, anos 1970 até início dos anos 1980 e a partir de meados dos anos 1990) é caracterizado por uma taxa de inflação com memória longa, mas não explosiva. Os autores concluem que as mudanças de regime inflacionário exercem efeitos sobre a memória de longo prazo da inflação, ou seja, no período mais recente, caracterizado por baixa inflação e baixa volatilidade, o coeficiente fracionário é menor que 1 .

Da Silva \& Leme (2011) utilizam a metodologia ARFIMA, assim como testes de raiz unitária com quebras estruturais, para examinar o IPCA, as expec- 
tativas de inflação e a taxa de juros real no Brasil, para o período compreendendo os meses de julho de 1999 a dezembro de 2010. Para o caso específico do IPCA brasileiro, o parâmetro ' $d$ ' encontrado foi de 0,43 , indicando que a inflação do país pode ser tomada como estacionária, com baixa persistência e reversão à média.

Em relação aos graus de persistências das inflações regionais, Caetano et al. (2009) utilizam testes de razão de variâncias para analisar os índices de preços ao consumidor (IPCA) de Belém, Fortaleza, Recife, Salvador, Belo Horizonte, Rio de Janeiro, São Paulo, Curitiba, Porto Alegre, Brasília e Goiânia. Para dados mensais de 1980 a 2009, os autores encontram um alto grau de persistência em todas as localidades analisadas, com exceção de Goiânia. Neste sentido, os componentes inerciais presentes nas séries regionais se mostraram significativos, com os efeitos de perturbações se dissipando de forma lenta.

\section{A Metodologia Econométrica dos Modelos ARFIMA e das Quebras Estruturais}

\subsection{Modelos ARFIMA ${ }^{2}$}

Tal como argumentado em Da Silva et al. (2011) e Da Silva \& Leme (2011), uma das maneiras de se analisar persistência de forma univariada é fazer uso dos diferentes testes de raiz unitária existentes na literatura. Nesse caso, o pesquisador estima a ordem de integração ' $d$ ' das séries, que pode assumir somente valores inteiros, seja $\mathrm{I}(0)$, se for estacionária, ou $\mathrm{I}(1)$, caso contrário. Outra possibilidade para se testar persistência univariada é fazer uso dos modelos ARFIMA (Auto-Regressive Fractionally Integrated Moving Average), como definido nos artigos de Granger \& Joyeux (1980) e Hosking (1981). Tais modelos são uma generalização dos modelos $\operatorname{ARIMA~}(p, d, q)$ com a vantagem de a ordem de integração ' $d$ ' poder ser fracionada entre 0 e 1 . Além disso, os modelos ARFIMA auxiliam na resolução do problema amplamente conhecido do baixo poder dos tradicionais testes de raiz unitária.

Com base em Hamilton (1994), um modelo ARMA (p, q) pode ser escrito da seguinte forma:

$$
y_{t}=c+\alpha_{1} y_{t-1}+\cdots+\alpha_{p} y_{t-p}+u_{t}+\beta_{1} u_{t-1}+\cdots+\beta_{q} u_{t-q}, t=1, \ldots, T
$$

onde ' $\alpha$ ' e ' $\beta$ ' são os coeficientes do modelo; ' $y$ ' uma variável genérica e ' $u$ ' representa o termo de erro com $u_{t}=N I D\left[0, \sigma^{2}\right]$.

Utilizando o operador defasagem:

$$
\left(1-\alpha_{1} L-\alpha_{2} L^{2}-\cdots-\alpha_{p} L^{p}\right) y_{t}=c+\left(1+\beta_{1} L+\beta_{2} L^{2}+\cdots+\beta_{q} L^{q}\right) u_{t}
$$

Dividindo-se ambos os lados pelo termo da esquerda: ${ }^{3}$

$$
y_{t}=\mu+\Phi(L) u_{t}
$$

onde:

\footnotetext{
${ }^{2}$ Esta parte está baseada em Da Silva et al. (2011) e Da Silva \& Leme (2011).

${ }^{3}$ Ver Hamilton (1994, p.59) para as condições necessárias para tal exercício.
} 


$$
\begin{gathered}
\Phi(L)=\frac{\left(1+\beta_{1} L+\beta_{2} L^{2}+\cdots+\beta_{q} L^{q}\right)}{\left(1-\alpha_{1} L-\alpha_{2} L^{2}-\cdots-\alpha_{p} L^{p}\right)} \\
\sum_{j=0}^{\infty}\left|\Phi_{j}\right|<\infty \\
\mu=\frac{c}{\left(1-\alpha_{1}-\alpha_{2}-\cdots-\alpha_{p}\right)}
\end{gathered}
$$

Dessa maneira, um processo integrado de ordem ' $d$ ' pode ter a seguinte representação:

$$
(1-L)^{d} y_{t}=\Phi(L) u_{t}
$$

Geralmente, assume-se que $d=1$, ou que a primeira diferença da série é estacionária. Todavia, valores fracionados de ' $d$ ' podem ser de grande utilidade.

Ainda segundo Hamilton (1994, p.448), considere a representação $M A(\infty)$ da equação (4). Se $d<0,5$, o inverso do operador $(1-L)^{-d}$ existe. Isso pode ser visto multiplicando-se ambos os lados da equação (4) por $(1-L)^{-d}$. O resultado é o seguinte:

$$
y_{t}=(1-L)^{-d} \Phi(L) u_{t}
$$

O operador $(1-L)^{-d}$ pode ser representado pelo seguinte filtro:

$$
(1-L)^{-d}=1+d L+\left(\frac{1}{2 !}\right)(d+1) d L^{2}+\left(\frac{1}{3 !}\right)(d+2)(d+1) d L^{3}+\cdots=\sum_{j=0}^{\infty} \lambda_{j} L^{j}
$$

onde $\lambda_{0} \equiv 1$ e:

$$
\lambda_{j}=\left(\frac{1}{j !}\right)(d+j-1)(d+j-2)(d+j-3) \cdots(d+1)(d)
$$

Pode-se demonstrar ${ }^{4}$ que se $d<1, \lambda_{j}$ pode ser aproximado para um grande " $j "$ por

$$
\lambda_{j} \cong(j+1)^{d-1}
$$

Assim, uma representação $M A(\infty)$, em que o coeficiente de impulso resposta $\lambda_{j}$ comporta-se, para grandes “ $j$ ”, como $(j+1)^{d-1}$, pode ser definido como:

$$
y_{t}=(1-L)^{-d} u_{t}=\lambda_{0} u_{t}+\lambda_{0} u_{t-1}+\lambda_{0} u_{t-2}+\cdots
$$

As autocorrelações das séries estacionárias ARMA podem ter um decréscimo exponencial, ao passo que séries fracionalmente integradas possuem decréscimos hiperbólicos. Em outras palavras, enquanto que os coeficientes de impulso-resposta de um processo ARMA estacionário desaparecem geometricamente, o processo da equação (8) se dissipa lentamente. Por conta dessa

\footnotetext{
${ }^{4}$ Ver Apêndice 15.A de Hamilton (1994).
} 
característica, processos fracionalmente integrados também são denominados de processos de memória longa.

Além disso, a sequência dos coeficientes MA limitantes $\left\{\lambda_{j}\right\}_{j=0}^{\infty}$ dados na equação (7) são "square-summable", desde que $d<0$, 5:

$$
=\sum_{j=0}^{\infty} \lambda_{j}^{2}<\infty \text { parad }<0,5
$$

Assim, para $d>0,5$, o processo deve ser diferenciado antes que fazer a descrição apresentada na equação (3) (Hamilton 1994).

Consequentemente, se $0 \leq d \leq 0,5$, a série é estacionária, com reversão à média e com um processo de memória longa. Se $0,5<d \leq 1$, a série não é estacionária mas permanece com reversão à média. Se $d \geqslant 1$, a série não é estacionária e não possui reversão à média (Gil-Alana 2001). Se $-0,5<d<0$, o processo é chamado de memória intermediária ou sobre-diferenciado.

Três métodos de estimação dos modelos ARFIMA são mais comuns: Máxima Verossimilhança Exata (Exact Maximum Likelihood - EML), Perfil Modificado de Verossimilhança (Modified Profile Likelihood - MPL) e Mínimos Quadrados Não-Lineares (Nonlinear Least Squares - NLS). Por definição, ambos EML e MPL impõem $-1<d<0,5$. O MPL é preferido em relação ao EML se o modelo incluir variáveis regressoras e se a amostra for pequena. A metodologia NLS permite que $d>-0,5$ e pode ser utilizada na estimação de séries não estacionárias (Baillie et al. 1996).

Uma vez que as séries examinadas parecem ser não estacionárias, a metodologia EML não se aplica porque ela é seriamente viesada para baixo para valores de ' $d$ ' próximos de 0,5 e maiores que 0,5. Dessa maneira, utilizaremos a metodologia NLS pois a mesma não sofre destes vieses usuais. O estimador NLS é baseado na maximização da seguinte função de verossimilhança:

$$
\ell_{N}(d, \Phi, \Theta)=-\frac{1}{2} \log \left(\frac{1}{T} \sum_{i=1}^{N} \widetilde{e_{t}}\right)
$$

onde os resíduos $\widetilde{e_{t}}$ são obtidos pela aplicação do Modelo $\operatorname{ARFIMA~}(p, d, q)$ aos $u_{t}$ e os vetores $\Phi$ e $\Theta$ representam, respectivamente, os parâmetros autorregressivos ' $p$ ' e os parâmetros de média móvel ' $q$ '.

Além disso, deve-se levar em conta que a persistência detectada pelos modelos ARFIMA pode estar sendo causada por quebras estruturais nas respectivas séries. Teoricamente, a importância de se analisar tal possibilidade está no trabalho de Diebold \& Inoue (2001), que argumentam que a literatura relacionada a processos de memória longa não presta a devida atenção à possibilidade de se confundir quebras estruturais e processos de memória longa. Granger \& Hyung (2004) também estudaram o mesmo problema e mostraram que a omissão de quebras ocasionais pode levar a uma superestimação do parâmetro ' $d$ '.

\subsection{Quebras Estruturais}

Além da investigação sobre a possibilidade de as séries possuírem propriedades de memória longa, também é necessário avaliar possíveis casos de quebras estruturais. A justificativa da relevância deste procedimento está no fato, já mencionado anteriormente, de se concluir que uma série possui certo grau 
de persistência quando, na verdade, são quebras estruturais influenciando o resultado.

A avaliação da ordem de integração das séries foi feita inicialmente por meio da utilização dos testes usuais de raiz unitária. No entanto, Perron (1989) destaca que testes, como o ADF, podem falhar em rejeitar uma falsa raiz unitária em função de problemas de especificação da tendência determinística. Perron $(1989,1997)$ e Zivot \& Andrews (1992) ampliaram o teste ADF ao considerar quebras exógenas e endógenas para evitar tal problema de especificação.

Clemente et al. (1998) propõem um teste de raiz unitária que lida com a possibilidade de ocorrência de duas quebras estruturais considerando dois tipos de eventos: outliers aditivos (AO) ou inovativos (IO). ${ }^{5}$ Os chamados eventos $\mathrm{AO}$ tratam de mudanças bruscas nas séries, ao passo que os eventos $\mathrm{IO}$ avaliam uma mudança gradual na média dos dados. Os autores expandem as estatísticas de Perron \& Vogelsang (1992) para o caso de duas mudanças na média. A ideia desses testes de raiz unitária com quebras estruturais é desenvolver um arcabouço para lidar com as limitações dos resultados empíricos anteriores, em que testes de raiz unitária não são capazes de rejeitar a hipótese nula (não estacionariedade). ${ }^{6}$

A hipótese nula testada por Clemente et al. (1998) é dada pela seguinte expressão:

$$
H_{0}: y_{t}=y_{t-1}+\delta_{1} D T B_{1 t}+\delta_{2} D T B_{2 t}+u_{t}
$$

A hipótese alternativa é dada por:

$$
H_{A}: y_{t}=\mu+d_{1} D U_{1 t}+d_{2} D U_{2 t}+e_{t}
$$

$D T B_{i t}$ representa uma $d u m m y$ de pulso que assume o valor 1 , se $t=T B_{i t}+1$, e o valor 0 , caso contrário. Tem-se ainda que $D U_{i t}=1$, se $t>T B_{i t}$, para $i=1,2$ e $D U_{i t}=0$, caso contrário, onde $T B_{1}$ e $T B_{2}$ são os períodos de tempo quando a média é modificada. Os autores assumem que $T B_{i t}=\lambda_{i} T$, para $i=1,2 \mathrm{com}$ $0<\lambda_{i}<1$ e $\lambda_{2}>\lambda_{1}$.

A hipótese de raiz unitária pode ser testada por meio da estimação da expressão na sequência para o caso em que as duas quebras fazem parte do outlier inovativo (IO):

$$
y_{t}=\mu+\rho y_{t-1}+\delta_{1} D T B_{1 t}+\delta_{2} D T B_{2 t}+d_{1} D U_{1 t}+d_{2} D U_{2 t}+\sum_{i=1}^{k} c_{i} \Delta y_{t-i}+e_{t}
$$

O próximo passo é obter o valor mínimo da pseudo razão-t para testar se o parâmetro autorregressivo ' $\rho$ ' é 1 para todas as combinações de quebras temporais. Para a derivação da distribuição assintótica desta estatística, Clemente et al. (1998) assumem que $0<\lambda_{0}<\lambda_{1}$ e $\lambda_{2}<1-\lambda_{0}<1$, implicando que o teste não é definido nos limites da amostra, sendo necessário escolher algum valor

\footnotetext{
${ }^{5}$ Ver Baum (2005) para outros detalhes do teste de Clemente et al. (1998).

${ }^{6}$ Ver Perron (1989), Banerjee et al. (1992), Christiano (1992), Zivot \& Andrews (1992), Perron (1997) e Vogelsang \& Perron (1998) como parte da literatura associada ao desenvolvimento dos testes de quebras estruturais para variáveis de tendência com quebras, além de Perron (1990) e Perron \& Vogelsang (1992) considerando os casos onde apenas uma mudança na média está presente. Lee \& Strazicich (2003) desenvolvem o teste de raiz unitária LM para duas quebras endógenas
} 
de aparas amostrais $\left(\lambda_{0}\right)$, além de impor que $\lambda_{1}$ e $\lambda_{2}$ assumam valores no intervalo $\left[(k+2) T, \frac{(T-1)}{T}\right]$. Os autores impõem uma restrição adicional, $\lambda_{2}>\lambda_{1}+1$, para eliminar casos de quebras temporais consecutivas. ${ }^{7}$

A hipótese nula de raiz unitária para testar a ocorrência de duas quebras estruturais, quando as mudanças são mais bem representadas como outliers aditivos $(\mathrm{AO})$, é desenvolvida em um procedimento de dois passos. O primeiro é remover a parte determinística da variável e estimar o seguinte modelo:

$$
y_{t}=d_{1} D U_{1 t}+d_{2} D U_{2 t}+\widetilde{y_{t}}
$$

O segundo passo é testar a hipótese de que $\rho=1$ e procurar o valor mínimo da estatística da razão-t no modelo abaixo:

$$
\widetilde{y_{t}}=\sum_{i=1}^{k} \omega_{1 i} D T B_{1 t-i}+\sum_{i=1}^{k} \omega_{2 i} D T B_{2 t-i}+\rho \widetilde{y}_{t-1}+\sum_{i=1}^{k} c_{i} \Delta \widetilde{y}_{t-i}+e_{t}
$$

onde $\widetilde{y_{t}}$ são os resíduos obtidos com a estimação da equação (15), DT $B_{i t}$ são as variáveis dummy incluídas na equação (16) para garantir que min $t_{\widehat{\rho}}^{A O}\left(\lambda_{1}, \lambda_{2}\right)$ convirja para a distribuição descrita na equação (4) de Clemente et al. (1998). ${ }^{8}$ A ideia é estimar uma regressão dos resíduos nos seus valores defasados, um número de diferenças defasadas e um conjunto de variáveis dummy necessárias para tornar a distribuição do teste estatístico tratável. ${ }^{9}$

\section{Os Dados}

Os dados utilizados na análise são as variações mensais dessazonalizadas do IPCA referente às regiões metropolitanas de Belém, Fortaleza, Recife, Salvador, Belo Horizonte, Rio de Janeiro, São Paulo, Curitiba e Porto Alegre, além de Brasília e do município de Goiânia. Comparações são feitas com o IPCA agregado nacionalmente e o período de análise compreende os meses de agosto de 1999 a dezembro de 2011.

A Tabela 1 reporta a estatística descritiva dos dados. Em termos de inflação média, o maior percentual é encontrado em Belém e o menor em São Paulo. Em termos de variabilidade, destacam-se o menor desvio padrão de Belo Horizonte e o maior de Brasília. Outro fato importante está relacionado às taxas máximas de inflação das regiões analisadas. Todas são referentes ao mês de novembro de 2002 e ligadas ao surto inflacionário no Brasil entre 2002 e 2003.

O caso brasileiro tem suas peculiaridades até por conta do passado de altas taxas de inflação verificadas no país, o que gerou enormes perdas de eficiência econômica e uma taxa de sacrifício muito grande cobrada de toda a população. A implementação do Plano Real, em 1994, aliviou o imposto inflacionário e provocou uma queda abrupta do índice de preços ao consumidor. Com a implantação do sistema de metas de inflação, o Brasil entrou em uma nova era na condução de sua política anti-inflacionária.

\footnotetext{
${ }^{7}$ Ver equação (4) de Clemente et al. (1998) para maiores detalhes da expressão para a distribuição da estatística do caso outlier inovativo (IO), representada por $\min _{\widehat{\rho}=0}^{I O}\left(\lambda_{1}, \lambda_{2}\right)$.

${ }^{8}$ Ver tabelas 1 e 2 de Clemente et al. (1998) para os valores críticos para amostras finitas e a distribuição assintótica das estatísticas IO e AO para 10000 replicações.

${ }^{9} \mathrm{O}$ valor da razão-t mínima é comparado com os valores críticos fornecidos por Perron \& Vogelsang (1992) dado que estas não seguem a distribuição padrão de Dickey-Fuller.
} 
Tabela 1: Estatística Descritiva: Taxas de Inflação IPCA (\% mês)

\begin{tabular}{lccccc}
\hline & Média & Mediana & Máximo & Mínimo & Desvio Padrão \\
\hline Brasil & 0,54 & 0,45 & 2,88 & $-0,03$ & 0,37 \\
Belo Horizonte & 0,57 & 0,47 & 2,60 & $-0,18$ & 0,38 \\
Brasília & 0,57 & 0,49 & 3,68 & $-0,45$ & 0,51 \\
Porto Alegre & 0,54 & 0,48 & 2,73 & $-0,29$ & 0,41 \\
Recife & 0,55 & 0,47 & 2,96 & $-0,30$ & 0,46 \\
Rio de Janeiro & 0,56 & 0,50 & 2,71 & $-0,25$ & 0,39 \\
Salvador & 0,56 & 0,46 & 3,06 & $-0,12$ & 0,47 \\
São Paulo & 0,51 & 0,43 & 2,79 & $-0,25$ & 0,40 \\
Belém & 0,59 & 0,48 & 3,14 & $-0,60$ & 0,46 \\
Curitiba & 0,54 & 0,48 & 3,12 & $-0,29$ & 0,46 \\
Goiânia & 0,56 & 0,51 & 3,51 & $-0,57$ & 0,48 \\
Fortaleza & 0,54 & 0,43 & 3,06 & $-0,50$ & 0,45 \\
\hline Fon
\end{tabular}

Fonte: IBGE.

As Figuras 1 e 2 auxiliam no entendimento da dinâmica recente do processo inflacionário brasileiro. Para isso, utilizamos a inflação acumulada em 12 meses, dado que facilita o olhar sobre a tendência do processo. Destacamse as elevações de preços no Brasil entre 2002 e 2003, uma consequência de incertezas geradas com o processo eleitoral para a Presidência da República. Em primeiro lugar, o mercado financeiro não conseguia ter uma clareza nas intenções do então futuro presidente. Segundo, o problema da dominância fiscal era claro e intensificado com aumentos de taxa de juros. Em terceiro lugar, a alta vulnerabilidade externa era também bem característica naquele momento. Sendo assim, a probabilidade de default não podia ser descartada, gerando depreciação cambial e, consequentemente, mais inflação (Bresser-Pereira \& Da Silva 2008).

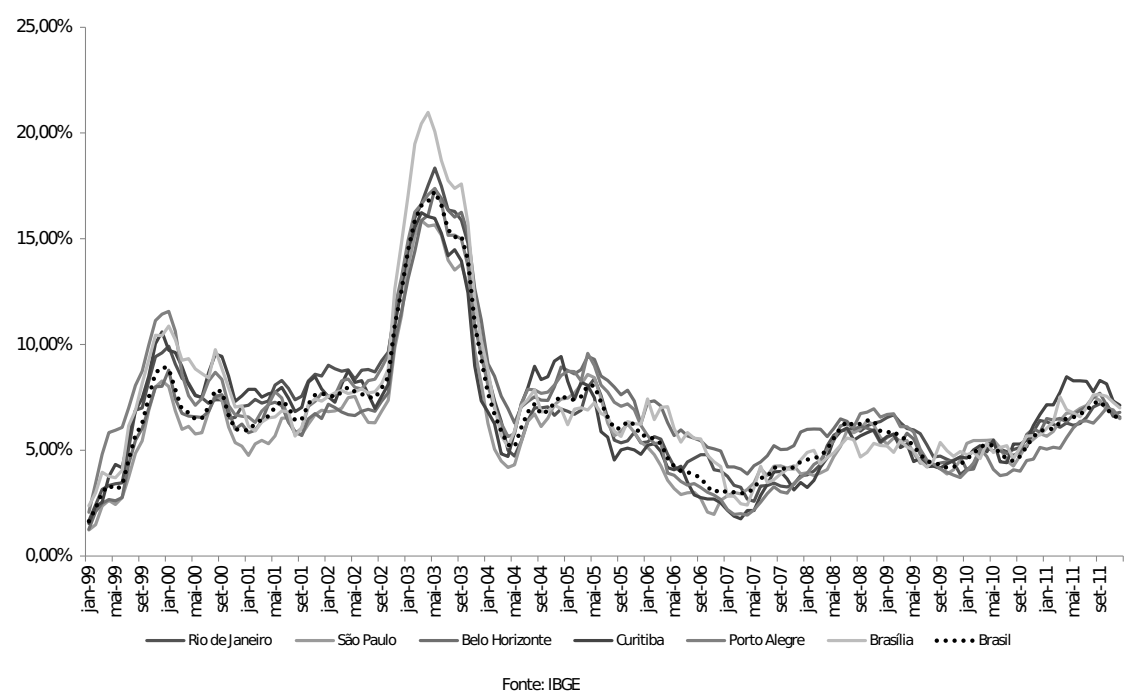

Figura 1: Taxas de Inflação IPCA (\% em 12 meses) 


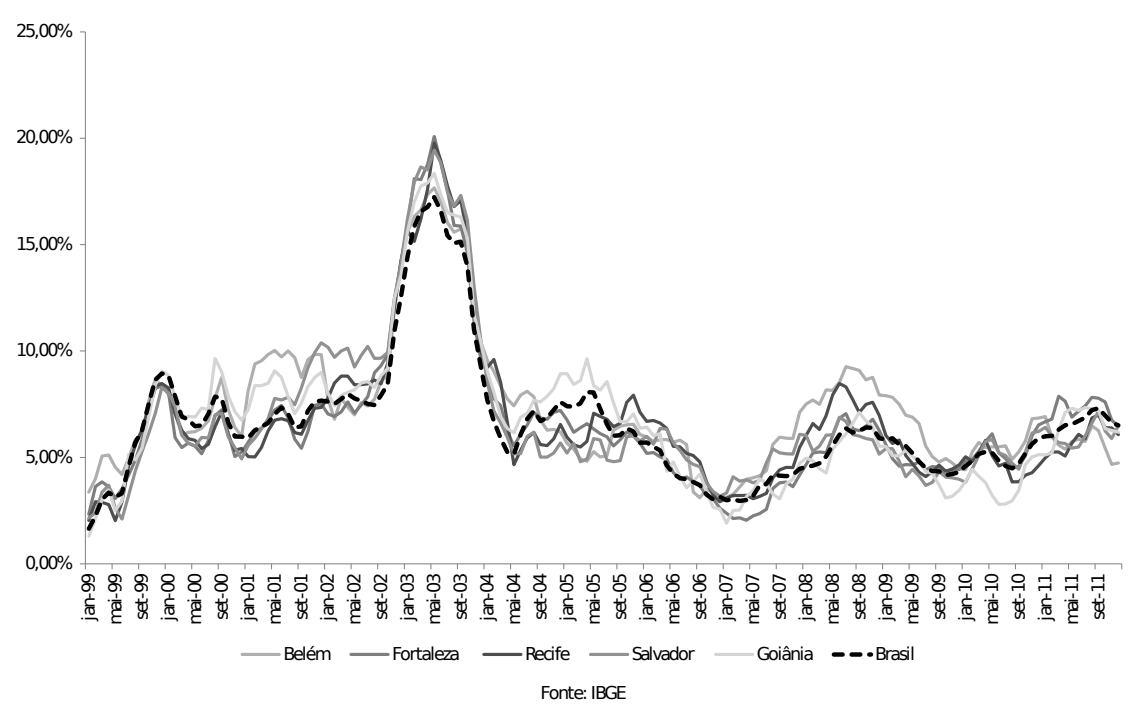

Figura 2: Taxas de Inflação IPCA (\% em 12 meses)

De todo modo, passado esse momento mais complicado da economia brasileira, as taxas de inflação começaram a ceder, sendo que o IPCA nacional tem conseguido se manter dentro dos limites determinados pelo sistema de metas de inflação, ainda que com dificuldades para cumprir o centro da meta. Mesmo assim, nota-se que algumas taxas regionais de inflação são persistentemente superiores ao IPCA nacional, enquanto outras são inferiores.

\section{Os Resultados}

\subsection{Testes Convencionais de Raiz Unitária}

Para efeito de comparação posterior, podemos iniciar a análise dos índices de inflação por intermédio da estimação dos testes de raiz unitária ADF, Phillips \& Perron (PP) e KPSS ${ }^{10}$ para todas as séries consideradas neste trabalho. Os resultados estão relatados na Tabela 2. Para os testes ADF e PP os resultados são categóricos: todas as séries apresentam estacionariedade $(d=0)$. Entretanto, Kwiatkowski et al. (1992) argumentam que testar raiz unitária como hipótese nula pode não ser aconselhável, pois a mesma é sempre aceita, ao menos que haja uma evidência contrária muito forte. A maioria dos dados econômicos pode não ter informação suficientemente capaz de decidir se os mesmos possuem ou não uma raiz unitária. Em outras palavras, os testes ADF e PP teriam um baixo poder. Assim, Kwiatkowski et al. (1992) propõem um teste de raiz unitária alternativo (KPSS) no qual a hipótese nula é a estacionariedade e a hipótese alternativa é raiz unitária.

O processo de estacionariedade somente é confirmado pelos testes KPSS, a $5 \%$ e 10\%, para Belo Horizonte, Fortaleza, Recife e São Paulo. Para as outras localidades, há algum tipo de ambiguidade nos resultados. Por isso, temos que dar um passo adiante e analisar as séries segundo Baillie et al. (1996), que argumentam que quando os testes KPSS rejeitam a hipótese nula de estacionariedade e a razão é uma integração fracionada, o teste PP também deveria

\footnotetext{
${ }^{10}$ Ver Dickey \& Fuller (1979), Kwiatkowski et al. (1992) e Phillips \& Perron (1988).
} 


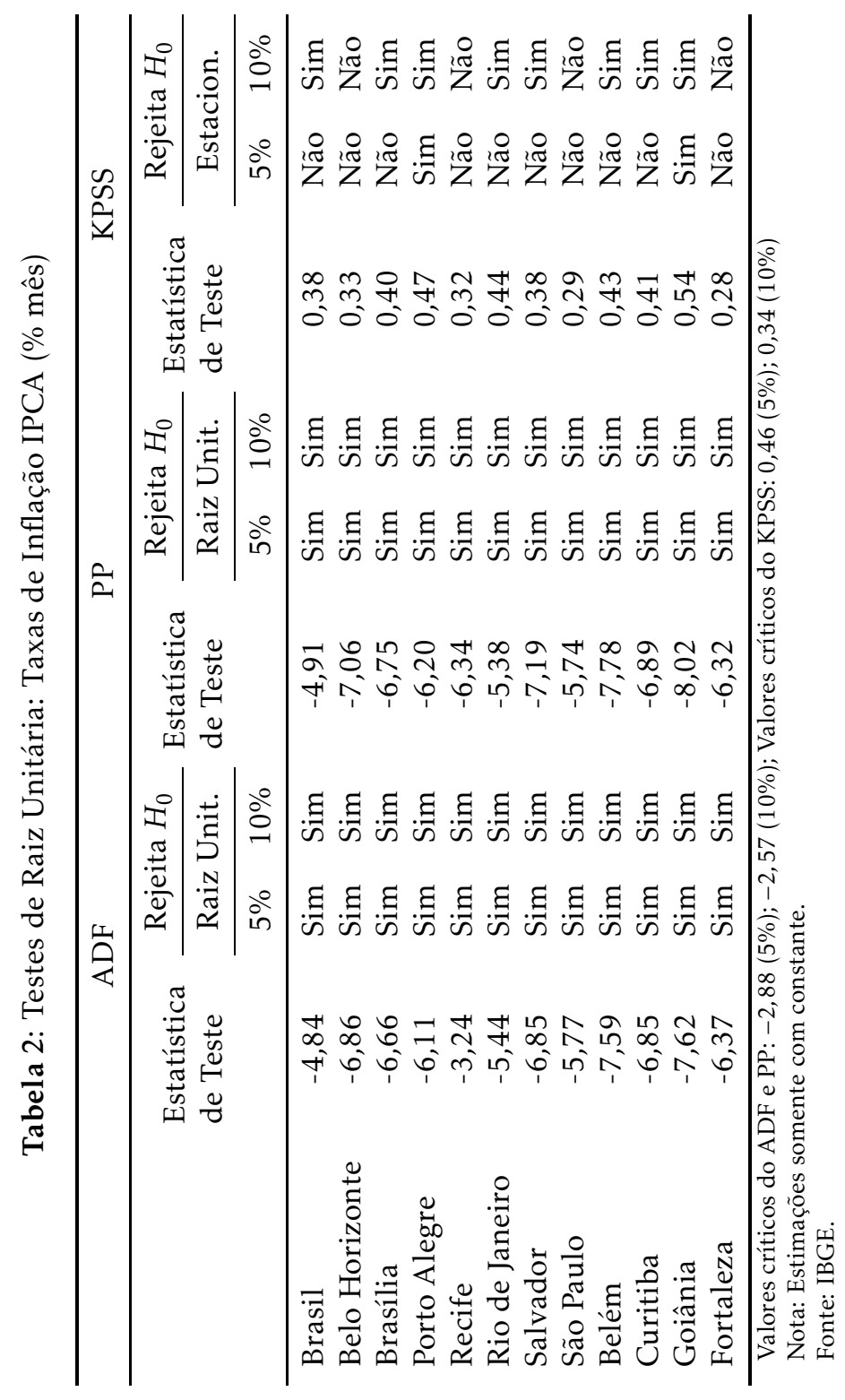


rejeitar a hipótese nula de raiz unitária. Isso aconteceu para a maioria das séries, pelo menos a $10 \%$ de significância. Quando há rejeição da nula do Teste PP e não rejeição da nula no KPSS, as chances são de que o processo seja $\mathrm{I}(0)$, sendo este os casos de Belo Horizonte, Recife, São Paulo e Fortaleza.

Além disso, apesar de nessa fase podermos chegar à conclusão de que a maioria das séries analisadas possui alguma característica de integração fracionada, existe ainda a possibilidade de que alguma confusão possa ser feita entre um processo de memória longa e um processo com quebras estruturais ocasionais. Analisar estas questões constitui nosso próximo passo.

\subsection{Testes de Raízes Unitárias com Quebras Estruturais}

A investigação sobre a ordem de integração das séries de tempo levando em conta a possibilidade de ocorrência de quebras estruturais foi desenvolvida a partir do teste de raiz unitária com duas quebras desenvolvido por Clemente et al. (1998), como definido na subseção 3.2.

Os resultados reportados na Tabela 3 sistematizam as informações referentes aos dois pontos de ocorrência das quebras estruturais para as taxas regionais de inflação, além da taxa nacional. A estatística do teste não rejeita a hipótese nula de raiz unitária para todas as localidades, com exceção de Porto Alegre. Os resultados dos testes de raiz unitária com quebras estruturais têm divergências em relação aos testes ADF, por exemplo, que chegaram à conclusão que todas as séries eram estacionárias, o que justifica a importância da análise feita.

Já os t-estatísticos para as duas quebras temporais $\left(D_{1 t}\right.$ e $\left.D_{2 t}\right)$ se mostraram estatisticamente significativos ao nível de $5 \%$ para todas as regiões, assim como para o Brasil, confirmando a ocorrência de duas quebras estruturais nas séries de taxa de inflação das localidades analisadas. A primeira quebra é primordialmente datada em agosto de 2002, com um caso no mês anterior e outro no mês posterior. Já a segunda quebra é menos concentrada, mas com maior ocorrência em março de 2003. O próximo passo é a incorporação destas quebras estruturais das séries de inflação e a investigação dos dados por meio dos Modelos ARFIMA.

\subsection{Modelos ARFIMA: Persistência nas Taxas Regionais de Inflação}

Em relação aos Modelos ARFIMA propriamente ditos, precisamos verificar se as taxas de decaimento ' $d$ ' das séries geram parâmetros mais próximos de 0 ou de 1. Para isso, o foco deve ser os modelos ARFIMA $(0, d, 0)$, isto é, sem os componentes AR ou MA. A Tabela 4 reporta as regressões referentes aos índices de preços ao consumidor de cada região sem os parâmetros AR ou MA. O IPCA brasileiro $(d=0,68)$ já demonstra certo controle da inércia inflacionária. No caso das inflações regionais, quase todos os parâmetros dos modelos $\operatorname{ARFIMA}(0, d, 0)$ são menores do que 0,5 . Isso caracteriza as séries como sendo estacionárias e com reversão à média de longo prazo. A exceção é São Paulo, cujo parâmetro é 0,57 . Belém e Goiânia apresentam as menores persistências entre as inflações regionais, com parâmetros em torno de 0,35. Além disso, para todos os modelos ARFIMA $(0, d, 0)$, a hipótese de que $d=0$ é rejeitada, assim como é rejeitada a hipótese de que $d=1$. Isso significa que a melhor estimação para a inflação regional brasileira pode ser um modelo fracionado. 
Tabela 3: Teste de Raiz Unitária com Duas Quebras Estruturais: Taxas de Inflação IPCA (\% mês)

\begin{tabular}{|c|c|c|c|c|}
\hline & $\mathrm{AR}(\mathrm{k})$ & Estatística de Teste & $1^{\text {a }} \underset{\text { (t-stat) }}{\text { Quebra }} D_{1 t}$ & $2^{\mathrm{a}} \underset{\text { (t-stat) }}{\text { Quebra }} D_{2 t}$ \\
\hline Brasil & 7 & $-3,27$ & $\begin{array}{l}\text { Ago-02 } \\
(10,24)^{*}\end{array}$ & $\begin{array}{l}\text { Mar-03 } \\
(-12,20)^{*}\end{array}$ \\
\hline Belo Horizonte & 8 & $-3,13$ & $\begin{array}{l}\text { Ago-02 } \\
(8,83)^{*}\end{array}$ & $\begin{array}{l}\text { Mar-03 } \\
(-9,63)^{*}\end{array}$ \\
\hline Brasília & 6 & $-5,25$ & $\begin{array}{l}\text { Ago-02 } \\
(9,50)^{*}\end{array}$ & $\begin{array}{c}\text { Fev-03 } \\
(-10,99)^{*}\end{array}$ \\
\hline Porto Alegre & 2 & $-6,55^{*}$ & $\begin{array}{l}\text { Ago-02 } \\
(8,01)^{*}\end{array}$ & $\underset{(-9,87)^{*}}{\operatorname{Mar}-03}$ \\
\hline Recife & 7 & $-3,87$ & $\begin{array}{l}\text { Set- } 02 \\
(7,32)^{*}\end{array}$ & $\begin{array}{l}\text { Mar-03 } \\
(-8,27)^{*}\end{array}$ \\
\hline Rio de Janeiro & 7 & $-3,36$ & $\begin{array}{l}\text { Ago-02 } \\
(9,42)^{*}\end{array}$ & $\underset{(-11,64)^{*}}{\operatorname{Mar}-03}$ \\
\hline Salvador & 9 & $-3,41$ & $\begin{array}{l}\text { Ago-02 } \\
(8,03)^{*}\end{array}$ & $\begin{array}{l}\text { Mar-03 } \\
(-9,90)^{*}\end{array}$ \\
\hline São Paulo & 10 & $-3,04$ & $\begin{array}{l}\text { Ago-02 } \\
(4,90)^{*}\end{array}$ & $\begin{array}{l}\text { Nov-02 } \\
(-5,37)^{*}\end{array}$ \\
\hline Belém & 6 & $-4,08$ & $\begin{array}{l}\text { Ago-02 } \\
(6,26)^{*}\end{array}$ & $\begin{array}{l}\text { Fev-03 } \\
(-7,55)^{*}\end{array}$ \\
\hline Curitiba & 11 & $-2,90$ & $\begin{array}{l}\text { Ago-02 } \\
(6,99)^{*}\end{array}$ & $\begin{array}{l}\text { Jan-03 } \\
(-8,26)^{*}\end{array}$ \\
\hline Goiânia & 7 & $-3,19$ & $\begin{array}{l}\text { Ago-02 } \\
(6,92)^{*}\end{array}$ & $\begin{array}{l}\text { Mar-03 } \\
(-8,54)^{*}\end{array}$ \\
\hline Fortaleza & 8 & $-3,29$ & $\begin{array}{l}\text { Jul-02 } \\
(8,44)^{*}\end{array}$ & $\begin{array}{l}\text { Fev-03 } \\
(-9,67)^{*}\end{array}$ \\
\hline
\end{tabular}

Sendo assim, seguimos o procedimento padrão e estimamos mais 15 modelos ARFIMA $(p, d, q)$ para cada série. Para isso, defasamos a parte autorregressiva (AR) e a parte média móvel (MA) até a terceira defasagem. Após a regressão dos 16 modelos para cada série, utilizamos o critério de informação de Schwarz para selecionar as defasagens mais apropriadas. A primeira característica a ser notada é que todos os parâmetros ' $d$ ' escolhidos estão próximos ou são menores que 0,5 . Para o IPCA brasileiro, o melhor modelo selecionado é um ARFIMA $(0,0,25,2)$, marcando o processo inflacionário do país como possuidor de baixa persistência, estacionário e com reversão à média de longo prazo.

\subsection{Integração Fracionária e Quebras Estruturais: O Procedimento de Granger \& Hyung}

Existe ainda a possibilidade de haver persistência sendo causada por quebras estruturais e isso gerar a possibilidade de sobrestimação do parâmetro ' $d$ ' devido à omissão dessas quebras. Esta pergunta pode ser respondida com o procedimento de Granger \& Hyung (2004) com o uso das datas das quebras selecionadas via teste de raiz unitária de Clemente et al. (1998) definidos na Tabela 3. O procedimento é baseado nos resíduos da seguinte regressão: 
Tabela 4: Modelos ARFIMA: Taxas de Inflação IPCA (\% mês)

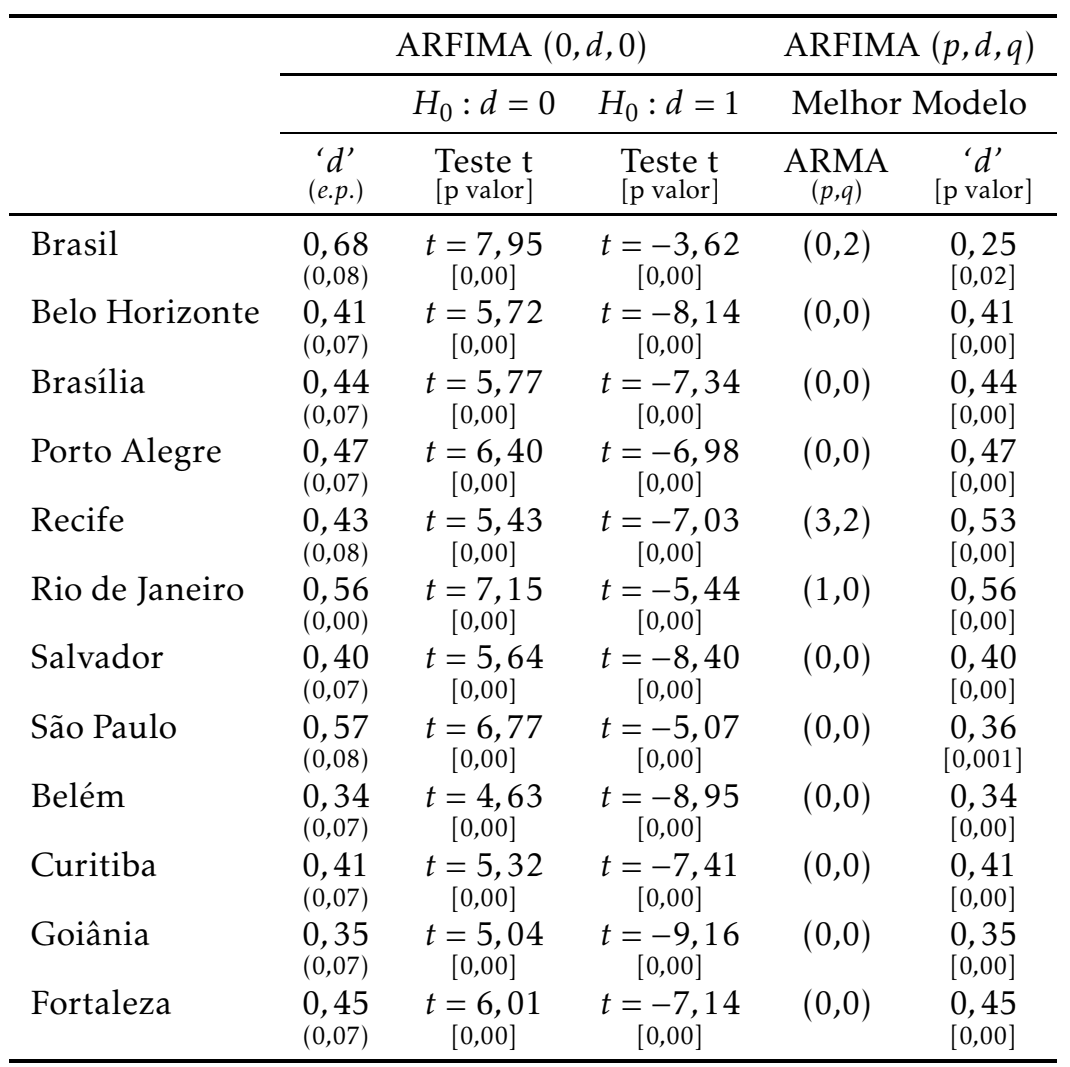

Fonte: IBGE.

$$
y_{t}=\beta^{\prime} Z_{t}+\xi_{t}
$$

onde $y_{t}$ representa uma das séries analisadas e $Z_{t}$ contém os termos determinísticos dos testes de raiz unitária com quebras.

Na sequência, foram estimados novos modelos ARFIMA $(0, d, 0)$ para todas as séries regionais de inflação. Como não foi utilizado nenhum componente autorregressivo nas estimações, espera-se que os parâmetros ' $d$ ' sejam próximos ou iguais a 1 se há presença de raiz unitária. Se o processo de memória longa for causado pela omissão de quebras estruturais, são esperados valores menores de ' $d$ ', refutando a memória longa anteriormente detectada. Os resultados reportados na Tabela 5 mostram que, para o caso brasileiro, o valor de $d=0,25$ é similar ao da Tabela 4, o que é um sinal da ausência de influência de quebras estruturais na análise.

Mas o intuito aqui é fazer uma análise do fenômeno inflacionário regional. Nesse caso, houve diferenças importantes. Para Belo Horizonte, o parâmetro encontrado foi de 0,06 , com possibilidade de ser zero pelo teste ' $t$ '. O resultado anterior era $d=0,41$, ou seja, houve influência da quebra. Isso significa que a persistência inflacionária é menor ainda para a capital mineira. O mesmo fenômeno foi encontrado para os testes de Rio de Janeiro, Brasília, Porto Alegre, Recife, Salvador, Belém, Fortaleza e Goiânia.

Por outro lado, os dados para a Região Metropolitana de São Paulo não se 
Tabela 5: Procedimento de Granger \& Hyung: ARFIMA $(0, d, 0)$ para os Resíduos

\begin{tabular}{lccc}
\hline & & $H_{0}: d=0$ & $H_{0}: d=1$ \\
\cline { 3 - 4 } & 'd' & Teste $t$ & $\begin{array}{c}\text { Teste } t \\
\text { [p valor] }\end{array}$ \\
\hline Brasil & 0,25 & 2,81 & $-8,33$ \\
Bplor] & $(0,09)$ & {$[0,00]$} & {$[0,0001]$} \\
Belo Horizonte & 0,06 & 0,81 & $-13,43$ \\
& $(0,07)$ & {$[0,42]$} & {$[0,0001]$} \\
Brasília & $-0,001$ & $-0,01$ & $-14,30$ \\
Porto Alegre & $(0,07)$ & {$[0,99]$} & {$[0,0001]$} \\
Recife & 0,18 & 2,41 & $-11,71$ \\
& $(0,07)$ & {$[0,01]$} & {$[0,0001]$} \\
Rio de Janeiro & 0,11 & 1,39 & $-11,13$ \\
& $(0,08)$ & {$[0,16]$} & {$[0,0001]$} \\
Salvador & 0,17 & 2,07 & $-10,38$ \\
& $(0,08)$ & {$[0,04]$} & {$[0,0001]$} \\
São Paulo & $-0,05$ & $-0,61$ & $-13,13$ \\
& $(0,08)$ & {$[0,53]$} & {$[0,0001]$} \\
Belém & 0,51 & 5,60 & $-5,44$ \\
& $(0,00)$ & {$[0,00]$} & {$[0,0001]$} \\
Curitiba & 0,036 & 0,474 & $-12,68$ \\
& $(0,07)$ & {$[0,63]$} & {$[0,0001]$} \\
Goiânia & 0,38 & 4,71 & $-7,75$ \\
& $(0,08)$ & {$[0,00]$} & {$[0,0001]$} \\
Fortaleza & 0,05 & 0,71 & $-13,57$ \\
& $(0,07)$ & {$[0,47]$} & {$[0,0001]$} \\
& 0,12 & 1,64 & $-12,57$ \\
\hline
\end{tabular}

Fonte: IBGE.

alteraram muito, marcando um parâmetro $d=0,51$, contra 0,36 na Tabela 4 . Isso mostra que as quebras não tiveram influência na análise para a referida região. O mesmo pode ser dito para o caso de Curitiba, cujo parâmetro ' $d$ ' registrado pelo método Granger \& Hyung foi 0,38, contra 0,41 anteriormente, sendo o mesmo estatisticamente significativo a $5 \%$ de probabilidade.

Vale a pena voltarmos nossa atenção para os casos de Recife e Rio de Janeiro novamente. Essas duas localidades apresentaram um parâmetro de persistência acima de 0,5 nos primeiros testes efetuados. Uma análise inicial poderia classificá-las como tendo taxas de inflação ainda com persistência considerável, de memória longa e com reversão à média no longo prazo. A aplicação do procedimento de Granger \& Hyung mostra que este não é o caso, pois fica detectada a influência das quebras na determinação do parâmetro de persistência. Em Recife, ' $d$ ' cai para 0,11 e no Rio ' $d$ ' cai para 0,17. Em suma, tanto a inflação nacional quanto as inflações regionais mostram características de estacionariedade e com reversão à média.

\section{Considerações Finais}

Este artigo investigou o fenômeno da persistência inflacionária do Índice de Preços ao Consumidor (IPCA) brasileiro das seguintes regiões metropolitanas e cidades: Belém, Belo Horizonte, Brasília, Curitiba, Fortaleza, Goiânia, Porto Alegre, Recife, Rio de Janeiro, Salvador, além do IPCA nacional. A análise 
considerou o período entre agosto de 1999 e dezembro de 2011 e a metodologia econométrica utilizada foi baseada nos modelos de memória longa, ou Autorregressivos de Integração Fracionária (ARFIMA), assim como em testes de raiz unitária com quebras estruturais.

Considerando as estimações feitas, pode-se concluir que testes usuais de raiz unitária geram resultados discrepantes sobre o comportamento da dinâmica inflacionária no Brasil e em suas principais regiões. Além disso, ao longo do período estudado, nota-se que a presença de quebras estruturais é comum em todas as séries analisadas, comprometendo a utilização de métodos simples de testes de estacionariedade. A possibilidade de as taxas regionais de inflação seguirem um processo de integração fracionária foi examinada por meio da aplicação dos modelos ARFIMA. Na primeira parte do estudo, o parâmetro ' $d$ ' obtido registrou média em torno de 0,40 , com resultados acima de 0,50 apenas para os casos do Rio de Janeiro e Recife. Significa que, na maioria dos casos, as persistências das taxas regionais de inflação no Brasil se mostraram controladas, sendo caracterizadas como estacionárias e com reversão a uma média de longo prazo. Quando as quebras estruturais foram devidamente tratadas, percebeu-se que as taxas de inflação do Rio e Recife também poderiam ser caracterizadas por terem baixa persistência e serem estacionárias.

Para efeito de comparação, Baillie et al. (1996) encontraram um parâmetro $d=0,59$, mas para um período bem mais longo e anterior ao sistema de metas de inflação (de 1957 a 1991). Reisen et al. (2003) também encontraram uma ordem de integração menor que 1 para o período de fevereiro de 1944 a fevereiro de 2000. Figueiredo \& Marques (2011) reportaram um parâmetro ' $d$ ' entre 0,72 e 0,93, também para um longo período (entre 1944 e 2009). Para uma análise levando-se em conta um período mais similar ao deste artigo, Figueiredo \& Marques (2009) indicaram um comportamento estacionário da taxa de inflação, podendo ser descrita como um processo fracionário de memória longa. Já Da Silva \& Leme (2011) chegaram a um parâmetro igual a 0,43 , ou seja, resultados bem próximos aos relatados neste trabalho para o caso do IPCA agregado.

Para efeito de comparação com números internacionais, Doornick \& Ooms (2004) reportaram um parâmetro igual a 0,32 para os EUA e $0,47<d<0,59$ para o Reino Unido. Já Gil-Alana (2005) encontrou $d=0,25$ para os EUA. Ambos os trabalhos também estão em linha com os nossos cálculos.

Em relação às inflações regionais, Caetano et al. (2009) analisaram praticamente três décadas de dados de inflação regional no Brasil, de 1980 a 2009. Dada essa extensão, os autores chegaram a um alto grau de persistência nas séries regionais, exceto para o município de Goiânia. Como focamos no período posterior à vigência do Plano Real, podemos observar que nossos resultados, ao relatarem taxas de inflação estacionárias e graus de persistência sob controle, vão em direção contrária aos resultados dos autores supracitados.

Em termos de política econômica, podemos dizer que, apesar de algumas diferenças entre os graus de persistência inflacionária das distintas regiões brasileiras, há uma clara conexão entre os valores regionais de inflação com o grau de persistência nacional, com um nítido processo de reversão à média de longo prazo. Significa que a condução de política monetária tem sido eficaz em evitar um comportamento inflacionário que gere graus de persistências elevados que poderiam colocar o país em trajetória inercial de inflação como em tempos pretéritos. 


\section{Agradecimentos}

Cleomar Gomes da Silva agradece ao CNPQ o apoio financeiro. Flávio Vilela Vieira agradece ao CNPQ e à FAPEMIG o apoio financeiro.

\section{Referências Bibliográficas}

Arida, P. \& Lara-Rezende, A. (1985), Inertial inflation and monetary reform in Brazil, in J. Williamson, ed., 'Inflation and Indexation: Argentina, Brazil and Israel', MIT Press.

Baillie, R. T., Chung, C. \& Tieslau, M. A. (1996), 'Analyzing inflation by the fractionally integrated ARFIMA-GARCH model', Journal of Applied Econometrics 11(1), 23-40.

Banerjee, A., Lumsdaine, R. \& Stock, J. (1992), 'Recursive and sequential tests of the unit root and trend break hypothesis: Theory and international evidence', Journal of Business and Economic Statistics 10, 271-287.

Batini, N. (2006), 'Euro area inflation persistence', Empirical Economics 31(4), 977-1002.

Batini, N. \& Nelson, E. (2001), 'The lag from monetary policy actions to inflation: Friedman revisited', International Finance 4(3), 381-400.

Baum, C. F. (2005), 'Stata: The language of choice for time-series analysis?', The Stata Journal 5, 46-63.

Bresser-Pereira, L. C. \& Da Silva, C. G. (2008), Inflation targeting in Brazil: A Keynesian approach, in L. R. Wray \& M. Forstater, eds, 'Keynes and Macroeconomics After 70 Years', Edward Elgar.

Bresser-Pereira, L. C. \& Nakano, Y. (1986), Inertial inflation and heterodox shocks in Brazil, in J. M. Rego, ed., 'Inertial Inflation, Theories of Inflation and the Cruzado Plan', Paz e Terra.

Caetano, S. M., Ferreira, D. M. \& Silva Jr, G. E. (2009), 'Dinâmica das inflações regionais brasileiras', Análise, Porto Alegre 20(2), 100-119.

Campêlo, A. K. \& Cribari-Neto, F. (2003), 'Inflation inertia and inliers: The case of Brazil', Revista Brasileira de Economia 57(4), 713-739.

Cati, R. C., Garcia, M. \& Perron, P. (1999), 'Unit roots in the presence of abrupt governmental interventions with an application to Brazilian data', Journal of Applied Econometrics 14(1), 27-56.

Christiano, L. (1992), 'Searching for breaks in GNP', Journal of Business and Economic Statistics 10, 237-250.

Clemente, J., Montañes, A. \& Reyes, M. (1998), 'Testing for a unit root in variables with double change in the mean', Economics Letters 59, 175-182.

Cribari-Neto, F. \& Cassiano, K. (2005), 'Uma análise da dinâmica inflacionária brasileira', Revista Brasileira de Economia 59(4), 535-566. 
Da Silva, C. G. \& Leme, M. C. S. (2011), 'An analysis of the degrees of persistence of inflation, inflation expectations and real interest rate in Brazil', Revista Brasileira de Economia 65, 289-302.

Da Silva, C. G., Lopes, D. T. \& Rebelo, A. M. (2011), 'Persistência inflacionária: Comparações entre três economias emergentes', Revista de Economia e Administração 10, 152-167.

Dickey, D. A. \& Fuller, W. A. (1979), 'Distribution of the estimators for autoregressive time series with a unit root', Journal of the American Statistical Association $74,427-431$.

Diebold, F. X. \& Inoue, A. (2001), 'Long memory and regime switching', Journal of Econometrics 105, 131-159.

Doornick, J. A. \& Ooms, M. (2004), 'Inference and forecasting for ARFIMA models, with an application to US and UK inflation', Studies in Nonlinear Dynamics and Econometrics 8(2).

Figueiredo, E. A. \& Marques, A. M. (2009), 'Inflação inercial como um processo de longa memória: Análise a partir de um modelo ARFIMA-FIGARCH', Estudos Econômicos 39(2), 437-458.

Figueiredo, E. A. \& Marques, A. M. (2011), 'Inflação inercial sob mudanças de regime: análise a partir de um modelo MS-ARFIMA, 1944-2009', Economia Aplicada 15(3), 443-457.

Fuhrer, J. \& Moore, G. (1995), 'Inflation persistence', Quarterly Journal of Economics 110(1), 127-159.

Gadzinski, G. \& Orlandi, F. (2004), 'Inflation persistence in the European Union, the Euro Area, and the United States', Eurosystem Inflation Persistence Network. European Central Bank Working Paper. No 414.

Gil-Alana, L. (2001), 'The persistence of unemployment in the USA and Europe in terms of fractionally ARIMA models', Applied Economics 33, 12631269.

Gil-Alana, L. (2005), 'Testing and forecasting the degree of integration in the US inflation rate', Journal of Forecasting 24, 173-187.

Granger, C. \& Hyung, N. (2004), 'Occasional structural breaks and long memory with an application to the S\&P 500 absolute stock returns', Journal of Empirical Finance 11, 399-421.

Granger, C. \& Joyeux, R. (1980), 'An introduction to long memory times series and fractional differencing', Journal of Time Series Analysis 1, 15-29.

Hamilton, J. (1994), Time Series Analysis, 1 edn, Princeton University Press.

Hosking, J. (1981), 'Modeling persistence in hydrological time series using fractional differencing', Water Resources Research 20, 1898-1908.

Kwiatkowski, D., Phillips, P. C. B., Schmidt, P. \& Shin, Y. (1992), 'Testing the null hypothesis of stationarity against the alternative of a unit root: how sure are we that economic time series are non stationary?', Journal of Econometrics $54,159-178$. 
Lee, J. \& Strazicich, M. C. (2003), 'Minimum LM unit root test with two structural breaks', The Review of Economics and Statistics 85, 1082-1089.

Ng, S. \& Perron, P. (2001), 'Leg length selection and the construction of unit root tests with good size and power', Econometrica 69, 1519-1554.

Perron, P. (1989), 'The great crash, the oil price shock, and the unit root hypothesis', Econometrica 57, 1361-1401.

Perron, P. (1990), 'Testing for a unit root in time series with a changing mean', Journal of Business and Economic Statistics 8, 153-162.

Perron, P. (1997), 'Further evidence on breaking trend functions in macroeconomic variables', Journal of Econometrics 355-385, 80.

Perron, P. \& Vogelsang, T. (1992), 'Nonstationarity and level shifts with an application to purchasing power parity', Journal of Business and Economic Statistics 10, 301-320.

Phillips, P. C. B. \& Perron, P. (1988), 'Testing for a unit root in time series regression', Biometrika 75, 335-346.

Reisen, V. A., Cribari-Neto, F. \& Jensen, M. (2003), 'Long memory inflationary dynamics: the case of Brazil', Studies in Nonlinear Dynamics and Econometrics 7, 1157-1173.

Tejada, C. \& Portugal, M. (2001), 'Credibilidade e inércia inflacionária no Brasil: 1986-1998', Estudos Econômicos 31(3), 459-494.

Vogelsang, T. \& Perron, P. (1998), 'Additional tests for a unit root allowing for a break in the trend function at an unknown time', International Economic Review 34(4), 1073-1110.

Yoon, G. (2003), 'The time series behavior of Brazilian inflation rate: New evidence from unit root tests with good size and power', Applied Economics Letters 10, 627-631.

Zivot, E. \& Andrews, D. W. K. (1992), 'Further evidence on the great crash, the oil-price shock and the unit root hypothesis', Journal of Business and Economic Statistics 10, 251-270. 